\title{
O Uso de Animações Interativas na Abordagem dos Conceitos da Energia Mecânica no Ensino Médio
}

\author{
The Use of Interactive Animations for Introducing the Concepts of Mechanical Ener- \\ gy in High School \\ Waltrudes Everton Sanches \\ Universidade Cruzeiro do Sul \\ Rua Galvão Bueno, 868 \\ Liberdade -São Paulo, SP \\ waltrudeseverton@hotmail.com \\ Juliano Schimiguel \\ Universidade Cruzeiro do Sul \\ Rua Galvão Bueno, 868 \\ Liberdade -São Paulo, SP \\ schimiguel@gmail.com \\ Mauro Sérgio Teixeira de Araújo \\ Universidade Cruzeiro do Sul \\ Rua Galvão Bueno, 868 \\ Liberdade -São Paulo, SP \\ mstaraujo@uol.com.br
}

\begin{abstract}
Resumo Este trabalho se propôs a investigar se o uso de animações interativas computacionais, na função de organizadores prévios, pode favorecer os processos de ensino e aprendizagem dos conceitos da Energia Mecânica. Para tanto, foi desenvolvida uma animação interativa, denominada Energia no Bate - estaca, e empregado um estudo que avaliou a influência da utilização desse recurso junto a 1650 alunos matriculados nos três turnos do Ensino Médio de uma escola pública estadual de São Luis. Foi utilizado como instrumentos de coleta de dados testes contendo questões que abordavam os conceitos da Energia Mecânica, envolvendo como variável dependente a média dos escores obtidos pelos grupos de alunos em cada etapa da pesquisa e como variável independente o emprego da animação interativa. Para verificar a influência da manipulação da variável independente sobre as médias dos escores dos grupos, foi usado o método da diferença ou plano clássico da prova das hipóteses. Os resultados obtidos sugerem que o uso de animações interativas como organizadores prévios é uma alternativa válida e eficaz para favorecer o processo ensino-aprendizagem dos conceitos da Energia Mecânica.
\end{abstract}

Palavras-Chave: Animação Interativa. Simulação. Energia. Ensino de Física.

\begin{abstract}
This study aimed to investigate whether the use of computational interactive animations in the function of previous organizers, may favor the processes of teaching and learning the concepts of Mechanical Energy. To that end, we developed an interactive animation, called Energia no Bate - estaca, and we employed a study that evaluated the influence of the use of this feature with the 1650 students enrolled in three shifts of high school in a public school in São Luis. It has been used as instruments of data collection tests containing questions that addressed the concepts of Mechanical Energy, involving as dependent variable the average scores obtained by groups of students in each stage of the research and as an independent variable employment of interactive animation. To check the influence of the manipulation of the independent variable on the mean scores of the groups, we used the difference method or plan of proof of classic hypotheses. The results suggest that the use of interactive animations as previous organizers is feasible and effective to facilitate the teaching-learning concepts of Mechanical Energy.
\end{abstract}

Keywords: Animation Interactive. Simulation. Energy. Teaching of Physics.

Recebido: 25 de Junho de 2013 / Aceito: 17 Agosto de 2013

DOI: 10.5753/RBIE.2013.21.02.01 


\section{Introdução}

Os problemas que afetam o ensino de Ciências em geral e de Física em particular não são recentes e tem merecido a atenção de diversos pesquisadores $[1,2,3]$, que apontam dificuldades relacionadas muitas vezes com as estratégias empregadas pelos docentes nos diferentes ambientes escolares.

No que se refere aos documentos oficiais, que fornecem sugestões e direcionamentos para a prática docente, é possível observar, por exemplo, nas Orientações Curriculares para o Ensino Médio (OCN) [4, p. 45] uma defesa explicita do uso de recursos e estratégias educacionais alternativas, enfatizando-se que "É importante que os métodos de ensino sejam modificados, capacitando o aluno a responder a perguntas e a procurar as informações necessárias, para utilizá-las nos contextos em que forem solicitadas".

Neste contexto em que estratégias alternativas devem ser colocadas em prática, trabalhos como Tavares e Santos [5], Balen e Netz [6] e Borcelli e Costa [7] e Costa et al. [8], entre muitos outros, apontam que o uso de recursos da informática como ferramentas didáticas, pode trazer significativos benefícios aos processos de ensino e aprendizagem em Ciências, em linha com o que estabelecem os Parâmetros Curriculares Nacionais $(\mathrm{PCN}+)[9, \mathrm{p}$. 111] ao afirmarem que "deve ser estimulado o uso adequado dos meios tecnológicos, como máquinas de calcular, ou das diversas ferramentas propiciadas pelos microcomputadores", uma vez que "essas estratégias permitem formas de representar e sistematizar o conhecimento que se confunde com a própria produção de um novo conhecimento". Desse modo, cabe destacar o posicionamento de Balen e Netz [6, p. 1], defendendo que:

Várias pesquisas têm demonstrado que novas tecnologias proporcionam a criação de ambientes que possibilitam a aprendizagem significativa dos conceitos associados ao conhecimento cientifico particularmente em Física e Química. As novas tecnologias provêem ferramentas que oportunizam a criação de ambientes de aprendizagem que estendam as possibilidades das tecnologias antigas. Uma ferramenta que tem demonstrado potencial expressivo como recurso para visualizar informações complexas é o microcomputador. O uso do computador possibilita aos estudantes dos diferentes níveis de ensino emergir em um ambiente virtual e interagir com modelos que possibilitam a aprendizagem significativa dos conceitos associados aos processos físico-químicos.

Esta pesquisa, na mesma linha dos trabalhos aqui citados, tem como objetivo central investigar as contribui- ções decorrentes do uso de ferramentas computacionais para os processos de ensino e aprendizagem de conceitos físicos, sendo norteada pela seguinte questão de investigação: De que maneira o uso de animações interativas computacionais, na função de organizadores prévios, pode favorecer os processos de ensino e aprendizagem dos conceitos relacionados com a Energia Mecânica?

As animações interativas, investigadas neste trabalho quanto a sua eficácia no papel de organizadores prévio, são, segundo Borcelli e Costa [7], filmes de computação gráfica que permitem ao usuário interagir, alterando determinados parâmetros, durante a simulação de um evento específico. Esses autores acrescentam ainda que este recurso seria capaz de permitir ao aluno "interagir, através da manipulação de variáveis que alteram o resultado final da simulação, possibilitando a visualização de situações que dificilmente seriam acessíveis em laboratórios didáticos" $[7$, p. 2].

Cabe destacar que os organizadores prévios constituem elementos presentes e discutidos no âmbito da teoria da Aprendizagem Significativa e, neste sentido, Rosa [10] explica que os conceitos subsunçores, aos quais os novos conceitos devem se conectar para que tenhamos uma aprendizagem significativa, vão sendo construídos ao longo do desenvolvimento do organismo que se encontra em constante interação com o meio. No entanto, no âmbito escolar, o professor não pode depender unicamente da formação espontânea desses elementos na estrutura cognitiva dos estudantes de modo a viabilizar a sua aprendizagem. Assim, uma solução para possibilitar a aprendizagem significativa de conteúdos onde os alunos ainda não reúnem os necessários subsunçores consiste na utilização dos Organizadores Prévios, que nas palavras de Rosa [10, p. 105] "[...] são uma tentativa de prover a ponte necessária entre a estrutura cognitiva atual e a estrutura do material instrucional que se está querendo ensinar". Por sua vez, Tavares e Santos [5, p. 3] definem o papel dos organizadores prévios como:

[...] um recurso proposto por Ausubel empregado para deliberadamente manipular a estrutura cognitiva dos alunos, a fim de que sejam desenvolvidos conceitos subsunçores, capazes de facilitar a aprendizagem significativa dos assuntos a serem ainda apresentados.

O destaque dado ao tema da energia neste trabalho justifica-se pelo fato do mesmo ser notoriamente relevante, além da própria Física, na Química e na Biologia, onde está presente na quase totalidade dos processos ali discutidos. A lei da conservação da energia, por exemplo, é apresentada por Feynman [11], como uma lei que governa todos os fenômenos naturais conhecidos até hoje. Kantor [12] acrescenta que não é possível imaginar uma única situação do cotidiano onde a energia não esteja 
presente. Também nos atuais documentos orientadores é destacada importância de se abordar o conceito de energia, como nas $\mathrm{OCN}[4$, p. 56], onde se estabelece que "No ensino da mecânica, por exemplo, os princípios de conservação dos movimentos e da energia são assuntos de grande relevância e merecem atenção principal".

Assim, a compreensão dos fundamentos básicos envolvidos no estudo da energia é imprescindível para que o aluno tenha o correto entendimento de muitos dos princípios que regem os fenômenos naturais, presentes inclusive em seu cotidiano, bem como dos processos utilizados no mundo produtivo.

\section{A Aprendizagem Significativa}

A aprendizagem significativa é o conceito central da teoria criada por David Paul Ausubel (1918-2008) [13], médico especializado em psiquiatria e professor da Universidade de Colúmbia, em Nova Iorque.

A aprendizagem significativa ocorre, segundo Ausubel [13], quando:

[...] idéias simbolicamente expressas sejam relacionadas de maneira substantiva (não literal) e não arbitrária ao que o aprendiz já sabe, ou seja, a algum aspecto de sua estrutura cognitiva especificamente relevante para a aprendizagem dessas idéias.

A primeira dessas duas condições, que se refere à necessidade dos novos conceitos serem incluídos de forma substantiva, Ausubel [13] explica que isto significa que o aluno deve apreender o sentido, o significado daquilo que se ensinou. Além disso, o novo conceito deve ser disposto de forma lógica e coerente na estrutura cognitiva do indivíduo [13]. Borcelli e Costa [7, p. 3] explicam que na aprendizagem significativa, "informações mais específicas são ligadas a conceitos mais gerais e inclusivos relacionados a esta informação. Estes conceitos préexistentes servem como ponto de ancoragem para a nova informação".

Os conceitos preexistentes na estrutura cognitiva do indivíduo são denominados por Ausubel [13, 15] como subsunçores, servindo de base para que novos conhecimentos sejam conectados. Quando o indivíduo incorpora novos conceitos sem que estes se liguem a um ou mais subsunçores, então, segundo Ausubel [15], se diz que está havendo aprendizagem mecânica, um processo freqüentemente observado em grande parte dos ambientes escolares, onde os alunos muitas vezes apenas procuram decorar alguns conceitos ou procedimentos algoritmos para resolverem questões propostas nas avaliações.

Neste tipo de aprendizagem as novas idéias não estabelecem uma relação lógica e coerente com os conceitos já presentes na estrutura cognitiva do indivíduo, mas são apenas armazenadas de forma arbitrária e desconexa. Quando o processo ocorre dessa forma, além de claro prejuízo para a longevidade do que é aprendido, em razão da falta de estabilidade da estrutura, o aprendizado não será substantivo, uma vez que é baseado apenas em simples memorização que tende a desaparecer em pouco tempo.

As OCN [4, p. 51] destacam a importância do professor considerar a base de conhecimentos prévios presentes nos alunos ao planejar e desenvolver suas atividades didáticas, afirmando que:

Os conhecimentos prévios dos alunos, e a exploração de suas contradições e limitações pelo professor, exigem que este elabore situações e problemas que o aluno não faria sozinho e que tenham o potencial de levar à aquisição de um conhecimento que o educando ainda não possui, mas que passará a ter significância dentro dos esquemas conceituais do aluno.

Por sua vez, Präss [14, p. 29], explicando as conseqüências da falta de substantividade da aprendizagem relacionada com o simples ato de se decorar os conteúdos, afirma que:

Como conseqüência dessa não flexibilidade (o aprendizado não é substantivo), o indivíduo não é capaz de expressar o novo conteúdo com linguagem diferente daquela com que este material foi primeiramente aprendido. De fato, ele não aprendeu o significado, o sentido do novo material, mas tão somente decorou a seqüência de palavras que o definia. Por conta disso, ele será incapaz de utilizar este conhecimento em contexto diferente daquele no qual fora primeiramente apresentado a estes conceitos/ idéias.

Apesar de Ausubel ter destacado as amplas vantagens da aprendizagem significativa em relação à mecânica, ele afirmava que no processo ensino-aprendizagem de determinados conteúdos havia circunstâncias em que a aprendizagem mecânica era necessária e inevitável.

Considerando os objetivos traçados para este trabalho, procuramos favorecer a formação de subsunçores na estrutura cognitiva dos alunos, viabilizando a aprendizagem, por meio da utilização dos Organizadores Prévios na forma de animações interativas computacionais.

Segundo Tavares e Santos [5], os organizadores prévios são materiais introdutórios que são apresentados antecipadamente e se caracterizam por apresentar um grau de generalidades e de inclusividade em um nível superior ao material principal que será apresentado em seguida. Esses organizadores, estruturados em diversos formatos como textos escritos, figuras, discussões, filmes 
ou outros, são empregados para "deliberadamente manipular a estrutura cognitiva dos alunos, a fim de que sejam desenvolvidos conceitos subsunçores, capazes de facilitar a aprendizagem significativa dos assuntos a serem ainda apresentados" [5, p. 3].

A teoria de Ausubel foi eleita como fundamentação deste trabalho, por apontar o distanciamento entre os conceitos presentes na estrutura cognitiva do aluno em relação ao que precisa aprender, como um dos principais problemas para que ocorra a aprendizagem significativa sobre um dado tema. Para atacar esse problema, Ausubel [13] propõe o uso de organizadores prévios que, a exemplo do que é feito em alguns trabalhos $[5,7]$, tem aqui o seu papel desempenhado por uma animação interativa elaborada para se abordar os conceitos da Energia Mecânica.

\section{Aspectos metodológicos da pesquisa}

Esta pesquisa foi desenvolvida no ano de $2011 \mathrm{em}$ uma escola da rede pública estadual de ensino de São Luis, capital do Maranhão. Foi considerado como a população, o conjunto composto pelos 1650 alunos, que segundo dados do INEP, foram matriculados em 2011 nessa escola, na $2^{\mathrm{a}}$ e $3^{\mathrm{a}}$ série do Ensino Médio, nos três turnos.

Os instrumentos de coleta de dados empregados nesta investigação foram baseados nos trabalhos de Tavares e Santos [5], Balen e Netz [6] e Borcelli e Costa [7], consistindo de testes que, no caso deste trabalho, abordam os conceitos da Energia Mecânica.

Foi tomada como variável dependente a média dos escores obtidos pelos grupos ao serem submetidos ao teste em cada etapa da pesquisa. Essa média, denotada por $\overline{\mathrm{X}}$, foi definida como a média aritmética dos escores $\mathrm{X}_{1}, \mathrm{X}_{2}, \mathrm{X}_{3} \ldots, \mathrm{X}_{\mathrm{N}}$, coletados na amostra. Desse modo, $\bar{X}=\frac{\sum_{j=1}^{N} X_{j}}{N}=\frac{X_{1}+X_{2}+X_{3}+\cdots+X_{N}}{N}$, onde $X_{1}, X_{2}, X_{3} \ldots, X_{N}$ representam cada um dos escores coletados junto à amostra. $\mathrm{O}$ número de valores de $\mathrm{X}$ é igual ao valor de $\mathrm{N}$, que representa o tamanho da amostra. Um escore $\mathrm{X}$ foi definido como um valor associado ao total de acertos de cada integrante do grupo pesquisado, ao se submeter ao teste.

O emprego da animação interativa foi tomado como a variável independente a ser manipulada. Para verificar a influência da manipulação da variável independente sobre as médias dos escores dos grupos, foi usado o método da diferença ou plano clássico da prova das hipóteses, o qual, de acordo com Lakatos e Marconi [16, p. 233], estabelece que:

Se em um caso, no qual aparece o fenômeno que se investiga e em outro caso, no qual não aparece, as circunstâncias são todas comuns, exceto uma, apresentando-se essa no primeiro, a circunstância única pela qual ambos os casos diferem é o efeito, ou a causa, ou uma parte indispensável do dito fenômeno.

Lakatos e Marconi [16] destacam que esse plano de prova das hipóteses exige dois grupos. Um deles é denominado experimental e é nele que manipularemos a variável independente. O outro, denominado grupo controle, passará por todas as etapas a que foi submetido o grupo experimental, excetuando-se apenas aquela onde a variável independente será manipulada. Assim, a partir da escolha ao acaso dos alunos na população em estudo, foram extraídas duas amostras: a primeira, denominada grupo experimental A, com 52 alunos, e a segunda, denominada grupo controle B, composto por 54 alunos, conforme mostra o Quadro 1:

\begin{tabular}{|c|c|c|c|}
\hline Turno & $\begin{array}{c}\text { Número } \\
\text { de } \\
\text { Alunos }\end{array}$ & Grupo & $\begin{array}{c}\text { Alunos/ } \\
\text { grupo }\end{array}$ \\
\cline { 1 - 2 } Matutino & 20 & \multirow{2}{*}{ Experimental A } & 52 \\
\cline { 1 - 2 } Vespertino & 19 & \multirow{2}{*}{ Controle B } & \multirow{2}{*}{54} \\
\cline { 1 - 2 } Noturno & 13 & & \\
\cline { 1 - 2 } Matutino & 15 & & \\
\cline { 1 - 2 } Vespertino & 27 & 12 &
\end{tabular}

Quadro 1 - Composição dos grupos de alunos.

Para fundamentar as conclusões sobre a validade do uso das animações, foram realizadas duas comparações entre as médias dos escores dos grupos pesquisados. A primeira foi um teste $t$ de Student para amostras pareadas. De acordo com Morcillo [17], duas amostras são pareadas quando os elementos do grupo estudado são comuns a ambas. Segundo esse autor, este estudo é geralmente aplicado ao grupo no qual a variável independente é manipulada e, de cada elemento dele, são obtidas duas medidas, ou seja, cada elemento é avaliado duas vezes; antes e depois da manipulação da variável independente. Este autor [17, p. 11] destaca ainda que "O objetivo fundamental no teste $t$ para amostras pareadas é avaliar o comportamento das diferenças observadas em cada elemento".

Para este teste, como desejávamos saber se a média dos escores do grupo experimental aumentou depois do emprego das animações, foram definidas as hipóteses:

$$
\begin{aligned}
& \mathrm{H}_{0}: \overline{\mathrm{X}}_{A_{\text {final }}}=\overline{\mathrm{X}}_{A_{\text {inicial }}} \\
& \mathrm{H}_{1}: \overline{\mathrm{X}}_{A_{\text {final }}}>\overline{\mathrm{X}}_{A_{\text {inicial }}}
\end{aligned}
$$

Os valores $\bar{X}_{A_{\text {inicial }}}$ e $\bar{X}_{A_{\text {final }}}$ representam, respectivamente, as médias dos escores do grupo experimental antes e depois do uso das animações interativas. 
A hipótese nula $\mathrm{H}_{0}$ assume que as médias dos escores do grupo experimental, antes e depois do uso das animações, ou são iguais ou a média final é menor que a média inicial desse mesmo grupo. A hipótese alternativa $\mathrm{H}_{1}$ contesta a hipótese inicial e assume que a média final do grupo experimental é maior que a inicial.

Para o julgamento das hipóteses, de acordo com Spiegel [18], primeiro devemos encontrar a estatística ou variável do teste $t_{c a l}$. Esse valor, calculado a partir das amostras, é usado na tomada de decisão sobre a aceitação ou rejeição das hipóteses. Segundo Guimarães [19], $t_{c a l}$ pode ser encontrado por meio da expressão:

$$
t_{c a l}=\frac{\bar{D}}{\left(\frac{S_{D}}{\sqrt{N}}\right)}
$$

O parâmetro $\bar{D}$ representa a diferença média entre os escores inicial e final, de cada elemento do grupo, ou seja, $\bar{D}=\frac{\sum D}{N}$. $D$ representa a diferença, para cada elemento do grupo, entre sua média inicial e final. $N$ representa o número de elementos da amostra e $S_{D}$ representa o desvio padrão das diferenças, que é uma medida do grau de dispersão das diferenças, ou seja, um indicador da variabilidade dos valores na amostra que se baseia nos desvios em torno da média $\bar{D}$. O desvio padrão das diferenças é dado por:

$$
S_{D}=\sqrt{\frac{\sum\left(D^{2}\right)-N \cdot \bar{D}^{2}}{N}}
$$

Conforme [18], além de calcular a estatística $t_{c a l}$, devemos escolher o nível de significância $\alpha$ do teste - parâmetro que Spiegel [18] define como a probabilidade de se cometer o erro de rejeitar $\mathrm{H}_{0}$ quando ela é verdadeira. Spiegel [18] esclarece também que, embora $\alpha$ seja uma escolha do pesquisador, normalmente são utilizados os valores $1 \%, 5 \%$ e $10 \%$. Optou-se neste trabalho por empregar um nível de significância de $5 \%$ para todos os testes de forma que tenhamos uma confiança de $95 \%$ de que a decisão sobre $\mathrm{H}_{0}$ não será equivocada. Guimarães [19] explica que o julgamento das hipóteses é feito comparando-se o valor de $t_{c a l}$ com o valor crítico do teste, representado por $t_{\alpha}$. Assim, quando a hipótese alternativa refuta $\mathrm{H}_{0}$ afirmando a superioridade de uma média em relação à outra, como é o caso deste primeiro teste, a única região de rejeição de $\mathrm{H}_{0}$ ficará a direita de $t_{\alpha} \mathrm{e} \mathrm{em}$ razão disso, diz-se que o teste é unilateral à direita. $\mathrm{O}$ valor crítico do teste $t_{\alpha}$ representa o limite da região de aceitação de $\mathrm{H}_{0}$, como pode ser visto na figura 1 .

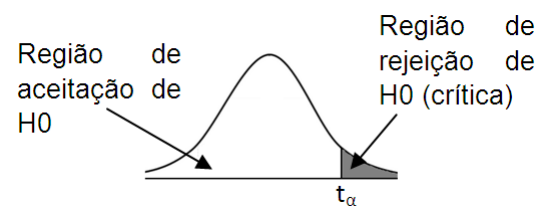

Figura 1 - Região de rejeição de H0 em teste unilateral.
Abordando a definição da região crítica e o julgamento das hipóteses, Guimarães [19, p. 132] esclarece que:

A faixa de valores da variável de teste que leva à rejeição de $\mathrm{H}_{0}$ é denominada região crítica (RC) do teste. A faixa restante constitui a região de aceitação (RA). Se o valor observado da estatística pertence a $\mathrm{RC}$, rejeitamos $\mathrm{H}_{0}$; caso contrário, não rejeitamos $\mathrm{H}_{0}$.

Em outras palavras, para o teste em questão, se $t_{c a l}>$ $t_{\alpha}$, dizemos que a variável do teste caiu na região crítica e dessa forma há evidências estatísticas para rejeitar $\mathrm{H}_{0}$; caso contrário, $\mathrm{H}_{0}$ será aceita. $t_{\alpha}$ é encontrado na tabela de distribuição de t, considerando o nível de significância $\alpha$ e o número de graus de liberdade GL. Segundo Guimarães [19], GL é um estimador da quantidade de informações independentes que serão utilizados no teste estatístico, podendo ser encontrado por $\mathrm{GL}=N-1$.

Para a segunda comparação, foi realizado um teste $t$ de Student para as médias de duas amostras independentes. Este teste é indicado quando desejamos comparar as médias de duas amostras extraídas de dois grupos distintos [19]. O objetivo deste teste é confrontar as médias finais do grupo experimental com as médias finais do grupo controle. Morcillo [17] enfatiza que a validade da comparação dos resultados finais dos dois grupos depende da constatação da igualdade das médias desses grupos, antes da manipulação da variável independente. Assim, a comparação das médias do grupo experimental com as médias do grupo controle ocorreu em dois momentos, antes e depois do uso das animações interativas. Para essas comparações devemos primeiro calcular o parâmetro $t$ de Student através da expressão [19]:

$$
t_{c a l}=\frac{\bar{X}_{A}-\bar{X}_{B}}{\sqrt{\frac{s_{A}^{2}}{N_{A}}+\frac{s_{B}^{2}}{N_{B}}}}
$$

$N_{A}$ e $N_{B}$ representam os tamanhos das amostras dos grupos A e B. $\bar{X}_{A}-\bar{X}_{B}$ é a diferença entre as médias dos escores dos grupos A e B. Os parâmetros $S_{A}$ e $S_{B}$ são os desvios padrão de cada grupo, os quais podem ser encontrados por:

$$
S=\sqrt{\frac{\sum(X-\bar{X})^{2}}{N-1}}
$$

Para a comparação entre as médias iniciais dos grupos A e $\mathrm{B}$, considerando que desejávamos testar a hipótese de que eram inicialmente iguais, foram construídas as hipóteses:

$$
\begin{aligned}
& \mathrm{H}_{0}: \overline{\mathrm{X}}_{\mathrm{A}_{\text {inicial }}}=\overline{\mathrm{X}}_{\mathrm{B}_{\text {inicial }}} \\
& \mathrm{H}_{1}: \overline{\mathrm{X}}_{\mathrm{A}_{\text {inicial }}} \neq \overline{\mathrm{X}}_{\mathrm{B}_{\text {inicial }}}
\end{aligned}
$$


De acordo com Guimarães [19], quando a hipótese alternativa refuta $\mathrm{H}_{0}$ afirmando que as duas médias são diferentes, o teste é bilateral (figura 2).

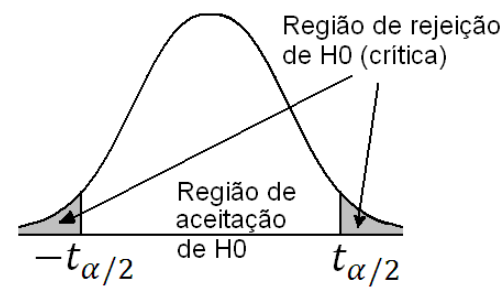

Figura 2 - Região de rejeição de H0 em teste bilateral. Fonte: Guimarães [19, p. 96] (adaptado)

Para o julgamento destas hipóteses, compara-se o valor de $t_{c a l}$ com o valor crítico do teste, representado por $t_{\alpha / 2}$. Neste teste, se $t_{c a l}>t_{\alpha / 2}$ ou $t_{c a l}<-t_{\alpha / 2}$, dizemos que a variável do teste caiu na região crítica e $\mathrm{H}_{0}$ será rejeitada. Por outro lado, caso $t_{c a l}$ fique compreendido entre $t_{\alpha / 2}$ e $t_{\alpha / 2}, \mathrm{H}_{0}$ será aceita. $t_{\alpha / 2}$ é encontrado na tabela de distribuição de t, considerando o nível de significância $\alpha$ e o número de graus de liberdade GL, que pode ser encontrado neste caso por $N_{A}+N_{B}-2$.

Para testar a afirmação de que a média final do grupo experimental $\mathrm{A}$ era superior à média final do grupo controle, foi aplicado o mesmo procedimento da comparação entre as médias iniciais, sendo que as hipóteses foram:

$$
\begin{aligned}
& \mathrm{H}_{0}: \overline{\mathrm{X}}_{\mathrm{A}_{\text {final }}}=\overline{\mathrm{X}}_{\mathrm{B}_{\text {final }}} \\
& \mathrm{H}_{1}: \overline{\mathrm{X}}_{\mathrm{A}_{\text {final }}}>\overline{\mathrm{X}}_{\mathrm{B}_{\text {final }}}
\end{aligned}
$$

Sendo este um teste unilateral, $\mathrm{H}_{0}$ é rejeitada caso tenhamos $t_{c a l}>t_{\alpha}$.

\section{Etapas de desenvolvimento da pes- quisa}

Como parte da metodologia empregada nesta investigação, optamos por desenvolver as atividades em quatro etapas, sendo que a primeira, a etapa inicial, teve a função de encontrar as médias dos escores dos grupos A e B, em seu estado inicial. Esses valores iniciais das médias dos escores dos dois grupos são necessários para que se possa estabelecer comparações com os resultados finais e, a partir disso, submeter as hipóteses ao plano clássico da prova das hipóteses descrito em [16] e apresentado anteriormente.

Nesta primeira etapa e na última, a exemplo do ocorre em [6], as questões foram corrigidas segundo o seguinte critério:

a) CT - Compreensão total. As respostas foram assim classificadas quando o aluno respondeu de acordo com o modelo aceito pelo formalismo da Física;

b) CP - Compreensão Parcial, quando a resposta foi parcialmente correta, apresentando falhas nos conceitos ou no tratamento matemático empregado;

c) CE - Concepção Errônea, quando o aluno respondeu utilizando argumentos inadequados ou quando o estudante não respondeu.

Com a finalidade de quantificar os resultados, definimos o escore $\mathrm{X}$ de cada participante como o resultado da expressão $X=1 \mathrm{~N}_{\mathrm{CT}}+0,5 \mathrm{~N}_{\mathrm{CP}}$, onde $\mathrm{N}_{\mathrm{CT}}$ e $\mathrm{N}_{\mathrm{CP}}$ representam os números de respostas classificadas como $\mathrm{CT}$ e $\mathrm{CP}$, respectivamente. Cabe destacar que $\mathrm{CT}$ vale $1,0, \mathrm{CP}$ vale 0,5 e CE vale 0 , valores definidos como a pontuação conferida para a parcela de respostas correta, ou seja, CT equivale a $100 \%$ correto, de modo que essa parcela corresponde a 1 , enquanto $\mathrm{CP}$ equivale a $50 \%$ correto, significando que essa parcela corresponde a 0,5 .

Para aplicação do teste, os alunos foram separados em três salas de aulas, independente do grupo ao qual pertenciam, e durante duas aulas de 50 minutos responderam às questões.

A segunda etapa desta pesquisa, aplicada somente ao grupo experimental A, consistiu no emprego de animações interativas como organizadores prévios, para introduzir conceitos sobre a Energia Mecânica. Nesta etapa, o grupo A primeiramente assistiu, no auditório da escola, a um pequeno vídeo caseiro ${ }^{1}$, com duração de 114 segundos, que mostra uma descida realizada em uma grande montanha russa. O propósito desse vídeo é despertar o interesse do aluno para o conteúdo que seria tratado posteriormente e com isso criar um ambiente onde ele se sinta mais motivado a se envolver com a atividade propostas, facilitando a conexão dos novos conceitos à sua estrutura cognitiva. A disposição do aluno para conectar os novos conceitos aos subsunçores é destacada por Ausubel [13] como um elemento indispensável para que ocorra a aprendizagem significativa. Deste modo a apresentação de vídeos, como o indicado aqui, assumiu um relevante papel no desenvolvimento deste trabalho. Em seguida, ainda no mesmo espaço físico, foi apresentada ao grupo a animação interativa utilizada nesta pesquisa: a Energia no Bate-estaca (figura 3), uma animação que desenvolvemos exclusivamente para o âmbito deste trabalho.

Essa animação, que aborda conceitos sobre Energia Mecânica, foi desenvolvida em linguagem Visual Basic (VB), no ambiente Visual Basic 6 da Microsoft. Nela podemos simular o processo de transformação de energia que ocorre em um bate-estaca, equipamento que em fun-

\footnotetext{
${ }^{1}$ Esse vídeo sobre a montanha russa pode ser encontrado no endereço http://www.youtube.com/watch?v=DFZ hve5K2s. Acesso em 25/04/2011.
} 
ção das várias construções em andamento em São Luís são vistos pelos alunos em diversos pontos da cidade, constituindo, portanto, um elemento de seu mundo vivencial.

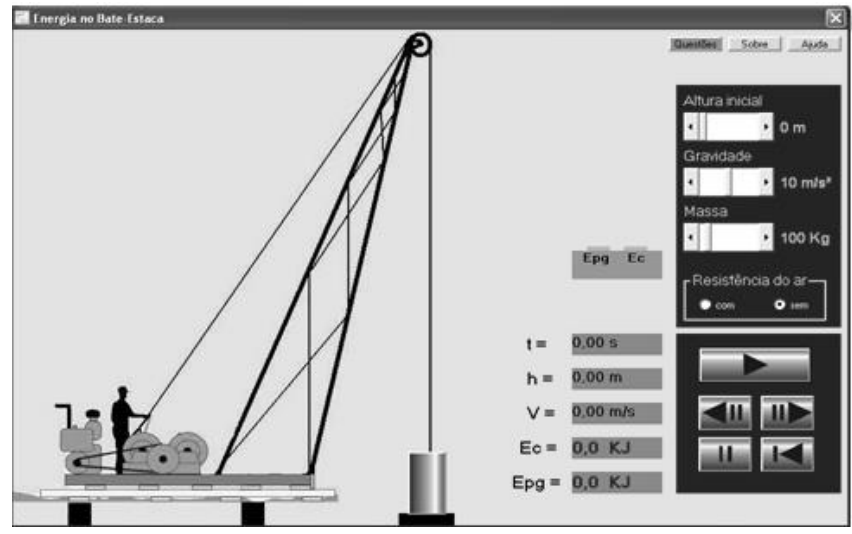

Figura 3 - Tela inicial da animação interativa Energia no Bate-Estaca.

Para as simulações, determinamos a altura da qual o martelo será abandonado, a aceleração da gravidade local, a massa do martelo e podemos decidir também se queremos considerar ou não a resistência do ar. Quando é iniciada a simulação, o aluno pode acompanhar a evolução temporal da altura e velocidade do martelo, assim como o valor da energia cinética e da energia potencial gravitacional disponível no sistema.

A opção pelo desenvolvimento de nossa própria animação decorreu do fato das opções disponíveis nos repositórios não apresentarem um grau de interatividade adequado para as condições que o trabalho demandava.

A exibição do vídeo da montanha russa e a apresentação da animação interativa ao grupo experimental ocorreram no mesmo dia e no mesmo local, utilizando para tanto, dois horários de 50 minutos.

Nos dois dias seguintes, utilizando os dois laboratórios de informática da escola durante dois horários de 50 minutos, os alunos, organizados em duplas, foram orientados a explorar a animação, Foi recomendado que iniciassem a exploração pela função Ajuda do programa (figura 4), que traz um roteiro mostrando como ajustar os parâmetros para produzir as simulações.

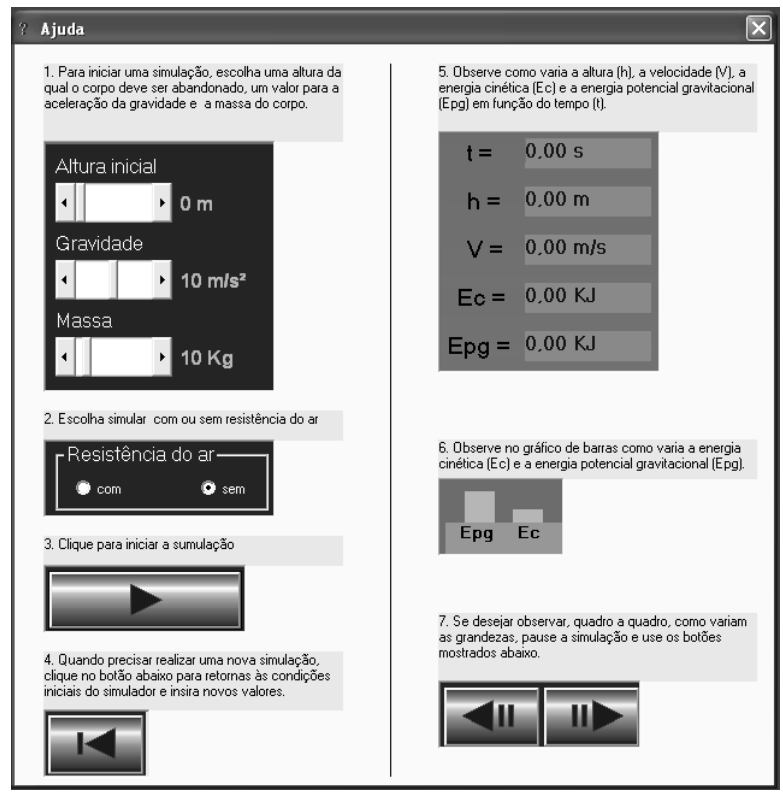

Figura 4 - Tela da ajuda da animação interativa Energia no BateEstaca.

Em seguida, o grupo foi estimulado a ler as questões que a animação propõe e resolve-las através de simulações realizadas no próprio programa. $\mathrm{O}$ formulário de questões da animação, acessado através de um dos botões do canto superior direito, contêm as seguintes perguntas:

1. Que tipo de energia o bloco ganha quando é levantado?

2. Essa energia depende do valor de quais grandezas?

3. Quando o bloco estiver parado a certa altura ele possuirá energia cinética $(\mathrm{Ec})$ ? Por quê?

4. Quando o bloco estiver parado a certa altura e aumentarmos sua massa, ocorrerá modificação em qual das energias?

5. Quando o bloco começar a cair, qual energia irá diminuir à medida que o bloco cai? Por que diminui?

6. À medida que o bloco cair, qual energia irá aumentar? Por que aumenta?

7. Se não considerarmos a resistência do ar, a soma das duas energias será sempre a mesma à medida que o corpo cai?

8. Se considerarmos a resistência do ar, a soma das duas energias será sempre a mesma à medida que o corpo cai?

Durante a apresentação e a exploração da animação não foi fornecida qualquer definição ou equação matemática sobre os conceitos ali envolvidos. Foi esclarecido que o aluno deveria descobrir as relações entre esses conceitos através de simulações feitas na própria animação. Foi 
recomendado que para responder as questões 1,2 e 4, o aluno precisava elevar o martelo do bate-estaca, manipulando a variável altura inicial, e, deixando-o parado a certa altura, observar o gráfico de barras. Com o martelo ainda parado a certa altura (figura 5), o aluno deveria modificar as variáveis gravidade e massa do martelo e observar as conseqüências disso no gráfico de barras e extrair as suas conclusões.

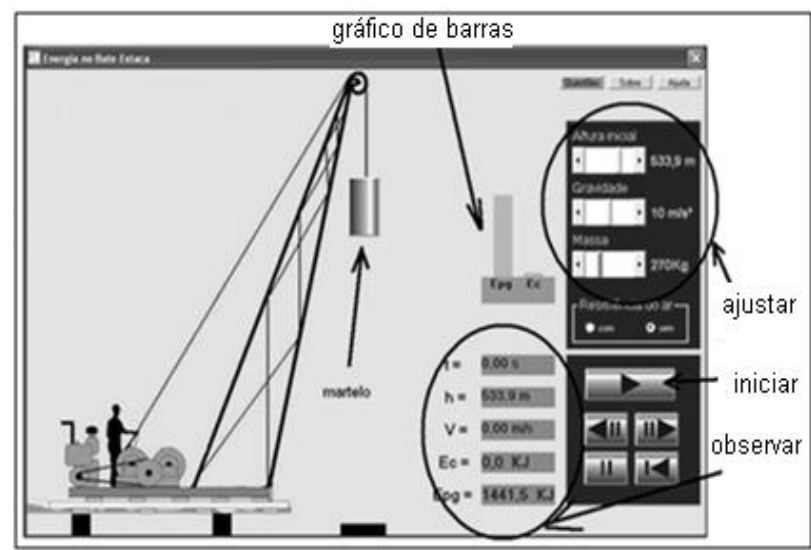

Figura 5 - Elementos da animação interativa Energia no Bate-Estaca.

Nas questões 3, 5 e 6, estando ainda o martelo parado a certa altura, o aluno deveria acionar o botão de início da simulação e observar no gráfico de barras e no campo das variáveis, o comportamento dos valores da altura, velocidade, energia cinética e energia potencial gravitacional. $\mathrm{O}$ aluno deveria observar qual energia cresce e qual decresce à medida que o corpo cai, e relacionando com a simulação anterior, concluir do que depende cada uma delas.

Para a questão 7, o aluno deveria colocar o martelo a certa altura e mantendo-o ali parado, anotar os valores da energia cinética e energia potencial gravitacional naquele ponto. Em seguida, acionar o início simulação, permitindo que o martelo caísse, e pausar o movimento em vários pontos, anotando os valores das duas energias em cada um deles. Para cada ponto, o aluno deveria somar as duas energias, encontrando a energia total em cada um deles e comparar os resultados. Inicialmente essa simulação deve ser realizada desconsiderando a resistência do ar, marcando esta opção na animação. Para a questão 8, o mesmo procedimento acima deveria ser realizado, porém, levando agora em consideração a resistência do ar.

$\mathrm{Na}$ terceira etapa, os alunos dos grupos A e B novamente foram separados em três salas, T1, T2 e T3, sendo que as duas primeiras foram compostas por 18 alunos do grupo B e 17 do grupo A, T3 foi formada por 18 alunos de cada grupo. Durante duas aulas de 50 minutos, em cada um dos dois dias destinados para essa etapa, os alunos receberam aulas expositivas sobre os conceitos da Energia Mecânica. As aulas foram ministradas nesses dois encontros por três professores de Física, usando como recursos apenas quadro branco e um projetor multimídia. As aulas foram ministradas segundo o roteiro abaixo.

\section{Energia e Trabalho}

1.1. Definições fundamentais

1.2. A Energia cinética

1.3. Teorema da energia cinética

1.4. Energia potencial

2. Conservação da energia

\subsection{Energia mecânica}

\subsection{Princípio da conservação da energia mecânica}

$\mathrm{Na}$ última etapa, os dois grupos $\mathrm{A}$ e $\mathrm{B}$ foram novamente submetidos ao mesmo teste da etapa inicial e os resultados do grupo A foram confrontados com os dados anteriores desse mesmo grupo e com os dados obtidos do grupo controle B nessa última etapa.

O resumo do cronograma de execução das etapas é mostrado no quadro 2.

\begin{tabular}{|c|c|c|c|c|}
\hline ETAPA & DESCRIÇÃO & DIA & GRUPO & DURAÇÃO \\
\hline $\begin{array}{l}1-\text { ETAPA } \\
\text { INICIAL }\end{array}$ & $\begin{array}{l}\text { Aplicação do } \\
\text { teste inicial }\end{array}$ & $1^{\circ}$ & $A \subset B$ & $\begin{array}{c}2 \mathrm{x} \\
50 \mathrm{~min}\end{array}$ \\
\hline \multirow{3}{*}{$\begin{array}{l}2-\text { USO DA } \\
\text { ANIMAÇÃO } \\
\text { INTERATIVA }\end{array}$} & $\begin{array}{l}\text { Vídeo montanha } \\
\text { russa }\end{array}$ & $2^{\circ}$ & A & $\begin{array}{c}2 \mathrm{x} \\
50 \mathrm{~min}\end{array}$ \\
\hline & \multirow{2}{*}{$\begin{array}{l}\text { Uso da animação } \\
\text { interativa }\end{array}$} & $3^{\circ}$ & A & $\begin{array}{c}2 \mathrm{X} \\
50 \mathrm{~min}\end{array}$ \\
\hline & & $4^{\circ}$ & A & $\begin{array}{c}2 \mathrm{x} \\
50 \mathrm{~min}\end{array}$ \\
\hline \multirow{2}{*}{$\begin{array}{l}3-\text { AULA } \\
\text { EXPOSITIVA }\end{array}$} & \multirow{2}{*}{$\begin{array}{l}\text { Aulas sobre os } \\
\text { conceitos da } \\
\text { energia mecânica }\end{array}$} & $5^{\circ}$ & $A \subset B$ & $\begin{array}{c}2 \mathrm{X} \\
50 \mathrm{~min}\end{array}$ \\
\hline & & $6^{\circ}$ & $A \in B$ & $\begin{array}{c}2 \mathrm{x} \\
50 \mathrm{~min}\end{array}$ \\
\hline $\begin{array}{l}4 \text { - ETAPA DE } \\
\text { VERIFICAÇÃO }\end{array}$ & $\begin{array}{l}\text { Aplicação do } \\
\text { teste de verifi- } \\
\text { cação }\end{array}$ & $7^{\circ}$ & $A \subset B$ & $\begin{array}{c}2 \mathrm{x} \\
50 \mathrm{~min}\end{array}$ \\
\hline
\end{tabular}

Quadro 2 - Etapas de desenvolvimento da pesquisa

\section{Análise dos Resultados Obtidos}

Nas tabelas 1 e 2 são mostrados os valores encontrados para a média dos escores, desvio padrão e variância para cada um dos dois grupos de alunos. Na tabela 1 esses parâmetros foram obtidos na etapa inicial da pesquisa, antes do emprego da animação interativa. Na tabela 2 , esses mesmos parâmetros foram obtidos na etapa de 
verificação, após a utilização da animação por parte do grupo experimental $\mathrm{A}$ e dos dois grupos assistirem às aulas expositivas.

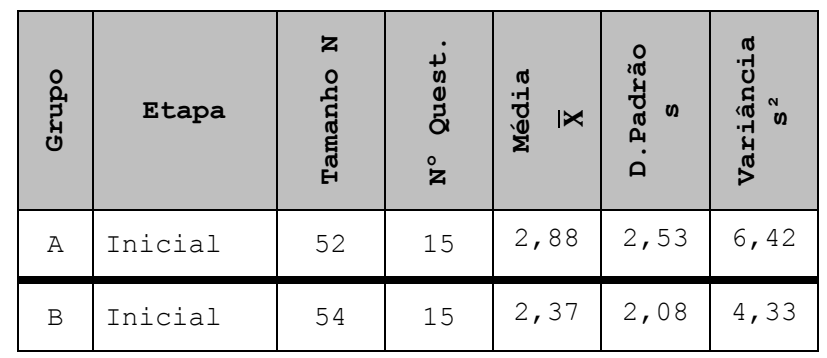

Tabela 1 - Grupos A e B, Etapa inicial, médias e dispersão.

\begin{tabular}{|c|c|c|c|c|c|c|}
\hline O̊. & Etapa & 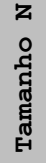 & 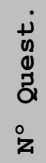 & 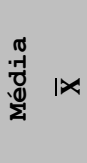 & 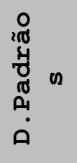 & 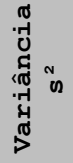 \\
\hline A & $\begin{array}{l}\text { Verifica- } \\
\text { ção }\end{array}$ & 52 & 15 & 5,02 & 2,79 & 7,81 \\
\hline B & $\begin{array}{l}\text { Verifica- } \\
\text { Ção }\end{array}$ & 54 & 15 & 3,62 & 2,71 & 7,34 \\
\hline
\end{tabular}

Tabela 2 - Grupos A e B, Etapa de Verificação, médias e dispersão.

Para o teste $t$ de Student envolvendo amostras pareadas, como já explicado anteriormente, testamos as hipóteses:

$$
\begin{aligned}
& \mathrm{H}_{0}: \overline{\mathrm{X}}_{A_{\text {final }}}=\overline{\mathrm{X}}_{A_{\text {inicial }}} \\
& \mathrm{H}_{1}: \overline{\mathrm{X}}_{A_{\text {final }}}>\overline{\mathrm{X}}_{A_{\text {inicial }}}
\end{aligned}
$$

O objetivo deste teste estatístico é identificar se a média dos escores do grupo experimental aumentou depois do emprego das animações interativas.

A estatística ou variável do teste $t_{c a l}$ foi encontrada através do procedimento de cálculo já descrito, obtendose $t_{c a l}=\mathbf{1 2 , 1 7 2}$. O valor crítico $\left(t_{\alpha}\right)$, encontrado na tabela de distribuição de $t$ (Anexo I), com base no nível de significância adotado e no número de graus de liberdade $\left(\mathrm{GL}=\mathrm{N}_{\mathrm{A}}-1\right)$, foi 1,645. Como constatamos que $t_{c a l}>t_{\alpha}$, concluímos que, como a variável do teste caiu na região crítica, há evidências estatísticas para rejeitar $\mathrm{H}_{0}$ e assumir que a média final do grupo experimental é superior a média inicial desse mesmo grupo.

Para comparação entre as médias iniciais dos grupos A e B, um teste $t$ de Student para as médias de duas amostras independentes foi empregado para avaliar as hipóteses:

$$
\mathrm{H}_{0}: \overline{\mathrm{X}}_{\mathrm{A}_{\text {inicial }}}=\overline{\mathrm{X}}_{\mathrm{B}_{\text {inicial }}}
$$

$$
\mathrm{H}_{1}: \overline{\mathrm{X}}_{\mathrm{A}_{\text {inicial }}} \neq \overline{\mathrm{X}}_{\mathrm{B}_{\text {inicial }}}
$$

Para o julgamento destas hipóteses, comparou-se o valor da variável do teste $t_{c a l}$ com o valor crítico, representado por $t_{\alpha / 2}$. Utilizando-se o procedimento de cálculo já descrito, encontrou-se $t_{c a l}=1,130$. Na tabela de distribuição de $t$ (Anexo I), considerando o nível de significância adotado e $N_{A}+N_{B}$ - 2 graus de liberdade, encontrou-se $t_{\alpha / 2}=1,960$. Assim, a região de rejeição de $\mathrm{H}_{0}$, região crítica, está fora do intervalo compreendido entre 1,960 e 1,960. Como $t_{c a l}$ ficou fora da região de rejeição de $\mathrm{H}_{0}$, podemos concluir que as médias iniciais dos escores dos dois grupos são iguais.

Finalmente, a comparação entre as médias finais dos grupos A e B, como já explicado anteriormente, foi realizada através de um teste $t$ de Student para as médias de duas amostras independentes que avaliou as hipóteses:

$$
\begin{aligned}
& \mathrm{H}_{0}: \overline{\mathrm{X}}_{\mathrm{A}_{\text {final }}}=\overline{\mathrm{X}}_{\mathrm{B}_{\text {final }}} \\
& \mathrm{H}_{1}: \overline{\mathrm{X}}_{\mathrm{A}_{\text {final }}}>\overline{\mathrm{X}}_{\mathrm{B}_{\text {final }}}
\end{aligned}
$$

A estatística $t_{c a l}$, calculada através do processo empregado no teste anterior, resultou $t_{c a l}=\mathbf{2 , 6 1 7}$. O valor crítico, encontrado na tabela de distribuição de $t$ (Anexo I), com base no nível de significância adotado e no número de graus de liberdade, $\mathrm{GL}=\mathrm{N}_{\mathrm{A}}-1$, foi $t_{\alpha}=\mathbf{1 , 6 4 5}$. Como o valor $t_{c a l}$ encontra-se além do limite $t_{\alpha}$, verificamos que a estatística do teste caiu na região de rejeição de $\mathrm{H}_{0}$. Assim, concluímos que há evidências estatísticas para aceitarmos que a média final do grupo experimental A é superior à média final do grupo controle $\mathrm{B}$, provavelmente em decorrência da utilização de recursos da informática caracterizados neste trabalho pelas animações interativas.

Cabe destacar que os resultados mostram que o avanço na média do grupo experimental foi superior ao observado no grupo controle. Assim, como as circunstâncias foram todas comuns, exceto a utilização das animações interativas pelo grupo A, apoiando-nos em Lakatos e Marconi [16], depreende-se que a circunstância única pela qual ambos os casos diferem concorreu para a produzir a diferença observada entre os avanços nas médias dos dois grupos.

\section{Conclusões}

O uso de recursos computacionais tem sido defendido por diversos pesquisadores $[5,6,7,8]$ e em linha com estas propostas, a busca de aprimoramento para os processos de ensino e aprendizagem de conceitos físicos justificou esta investigação envolvendo a utilização de simulações interativas com alunos do Ensino Médio de uma escola pública de São Luis, capital do Maranhão.

Os resultados dos testes mostram, além do avanço da 
média dos escores do grupo experimental, um avanço desta mesma variável também no grupo controle. Este efeito já era esperado visto que os dois grupos assistiram a aulas expositivas sobre o assunto. Em relação a este cenário, Campbell e Stanley [20] alertam que a ação de eventos específicos, distintos da manipulação da variável experimental, pode comprometer a validade do estudo já que seus efeitos podem ser confundidos com os efeitos da variável experimental. Porém, segundo esses mesmos autores, caso os eventos estranhos à variável experimental sejam comuns aos dois grupos, essa variável é controlada pelo método na medida em que a variação na média do grupo experimental, produzida por esta variável, também será encontrada no grupo controle. Assim, Lakatos e Marconi [16] afirmam que podemos supor que a diferença entre os escores antes e depois, no grupo de controle, seja o resultado da ação das variáveis estranhas. Já no grupo experimental, a diferença entre os escores antes e depois, corresponde à ação da variável experimental adicionada aos efeitos das mesmas variáveis estranhas que atuaram também sobre o grupo de controle. Deste modo, como os resultados mostraram um avanço na média do grupo experimental superior ao do grupo controle, e o processo ao qual foram submetidos diferiu pelo fato de somente o grupo experimental ter passado pela etapa de utilização das animações interativas, é possível concluir por meio dos resultados dos testes estatísticos empregados, a um nível de significância de $5 \%$, que o uso das animações interativas concorreu para produzir o avanço na média do grupo experimental.

Evidentemente, outras variáveis como as diferenças de motivação dos grupos, que não são consideradas ou isoladas nesta metodologia, podem trazer implicações importantes aos resultados da pesquisa. Ainda assim, comparando os resultados observados neste trabalho e que corroboram com os obtidos por outros pesquisadores em investigações desenvolvidas e mencionadas aqui, consideramos bastante plausível concluir que as animações interativas, na função de organizadores prévios, podem favorecer os processos de ensino e de aprendizagem dos conceitos da Energia Mecânica que foram explorados ao longo deste trabalho.

Ao possibilitarmos que os estudantes se envolvessem com ferramentas computacionais interativas e pudessem manipular e testar os parâmetros físicos envolvidos acreditamos estar proporcionado meios para que pudessem construir novos conhecimentos e, com isso, ampliar a sua capacidade de atuação autônoma diante das situações exploradas. Neste sentido, Mizukami [21, , p. 73] destaca que:

A escola, dessa forma, deveria dar a qualquer aluno a possibilidade de aprender por si próprio, oportunidades de investigação individual, possibilitando-lhe todas as tentativas, todos os tateios, en- saios que uma atividade real pressupõe. Isso implica diretamente que a motivação não venha de fora, mas lhe seja intrínseca, ou seja, da própria capacidade de aprender, para que se torne possível a construção de estruturas do ponto de vista endógeno.

Considerando a importância do tema abordado para o ensino de Ciências, julgamos que merece ser considerada a possibilidade de desenvolvimento de trabalhos semelhantes empregando outros recursos computacionais com uma orientação teórica diferente, como tutoriais ou jogos, para que se possa ampliar a base de dados e os conhecimentos obtidos, fortalecendo a convicção de que estes recursos computacionais podem efetivamente contribuir para a aprendizagem dos estudantes.

\section{Referências}

[1] C. W. Rosa, Á. B. Rosa. Ensino da Física: tendências e desafios na prática docente. Revista Iberoamericana de Educación, 42 (7), 1-12, 2007.

[2] Carlos Fiolhais; Jorge Trindade. Física no computador: o computador como uma ferramenta no ensino e na aprendizagem das ciências físicas. Revista Brasileira de Ensino de Física, 25 (3), 259-272, 2003.

[3] F. Marineli; J. L. Pacca. Uma interpretação para dificuldades enfrentadas pelos estudantes em um laboratório didático de Física. Revista Brasileira de Ensino de Física, 28 (4), 497-505, 2006.

[4] BRASIL. Orientações Curriculares para o Ensino Médio (OCN); volume 2. Ciências da Natureza, Matemática e suas Tecnologias. Secretaria de Educação Básica. Brasília: Ministério da Educação, Secretaria de Educação Básica, 135 p., 2006.

[5] R. Tavares; J. N. D. Santos. Organizador prévio e animação interativa. In Anais do IV International meeting on meaningful learning. Maragogi, 2003.

[6] O. Balen; P. A. Netz. Utilizando a modelagem e a simulação computacional no estudo do comportamento dos gases. In Anais do XVI Simpósio Nacional de Ensino de Física, p. 1-4, 2005.

[7] A. F. Borcelli; S. S. C. D. Costa. Animação Interativa: um material potencialmente significativo para a aprendizagem de conceitos em física. In Anais do XI Encontro de Pesquisa em Ensino de Física. Curitiba: [s.n.]. 2008. 
[8] H. R. Costa; Silva, A. L. P.; Lima, J. B.; Varela Júnior, J. J. G. Desenvolvimento e aplicação de um software educacional na aprendizagem significativa do estudo dos gases. In Anais do $50^{\circ}$ Congresso Brasileiro de Química. Cuiabá: CBQ. 2010.

[9] BRASIL. PCN+ Ensino Médio: Orientações Educacionais Complementares aos Parâmetros Curriculares Nacionais. Vol. Ciências da natureza, Matemática e suas Tecnologias. Brasília: MEC/Semtec, 2002.

[10] P. R. D. S. Rosa. Instrumentação para o ensino de ciências. Editora UFMS, Campo Grande, 2010.

[11] Richard Feynman. Física em seis lições. 2.ed. Ediouro, Rio de Janeiro, 1999.

[12] C. A. Kantor et. al. Física $1^{\circ}$ Ano (coleção Quanta Física; v.1). $1^{a}$ Edição. Editora PD, São Paulo, 2010.

[13] D. P. Ausubel; J. D. Novak; J. Hanesian. Psicologia Educacional. Interamericana, Rio de Janeiro, 1980.

[14] A. R. Präss. Teorias da aprendizagem. Porto Alegre. 2008.

[15] D. P. Ausubel. Aquisição e retenção de conhecimento: uma perspectiva cognitiva. Plátano, Lisboa, 2003.

[16] Eva Maria Lakatos; Marina de Andrade Marconi. Metodologia Científica. 5. ed. Atlas, São Paulo, 2008.

[17] A. M. Morcillo. Teste t Student. Campinas: Universidade Estadual de Campinas - Faculdade de Ciências Médicas, 2004.

[18] M. R. Spiegel. Estatística. 2. ed. Mcgraw-Hill, São Paulo, 1985.

[19] P. R. B. Guimarães. Métodos Quantitativos Estatísticos. IESDE Brasil S.A., Curitiba, 2008.

[20] D. T. Campbell; J. C. Stanley, Delineamentos experimentais e quase-experimentais de pesquisa. EPU/Edusp, São Paulo, 1979.

[21] M. G. N. Mizukami. Ensino: as abordagens do processo. Temas Básicos de Educação e Ensino. E.P.U., São Paulo, 119. p, 1986. 